\title{
Dynamics of a large system of spiking neurons with synaptic delay
}

\author{
Federico Devalle, ${ }^{1,2,{ }^{*}}$ Ernest Montbrió, ${ }^{1}$ and Diego Pazó ${ }^{3}$ \\ ${ }^{1}$ Neuronal Dynamics Group, Department of Information and Communication Technologies, \\ Universitat Pompeu Fabra, 08018 Barcelona, Spain \\ ${ }^{2}$ Department of Physics, Lancaster University, Lancaster LA1 4YB, United Kingdom \\ ${ }^{3}$ Instituto de Física de Cantabria (IFCA), CSIC-Universidad de Cantabria, 39005 Santander, Spain
}

(Received 21 August 2018; published 22 October 2018)

\begin{abstract}
We analyze a large system of heterogeneous quadratic integrate-and-fire (QIF) neurons with time delayed, all-to-all synaptic coupling. The model is exactly reduced to a system of firing rate equations that is exploited to investigate the existence, stability, and bifurcations of fully synchronous, partially synchronous, and incoherent states. In conjunction with this analysis we perform extensive numerical simulations of the original network of QIF neurons, and determine the relation between the macroscopic and microscopic states for partially synchronous states. The results are summarized in two phase diagrams, for homogeneous and heterogeneous populations, which are obtained analytically to a large extent. For excitatory coupling, the phase diagram is remarkably similar to that of the Kuramoto model with time delays, although here the stability boundaries extend to regions in parameter space where the neurons are not self-sustained oscillators. In contrast, the structure of the boundaries for inhibitory coupling is different, and already for homogeneous networks unveils the presence of various partially synchronized states not present in the Kuramoto model: Collective chaos, quasiperiodic partial synchronization (QPS), and a novel state which we call modulated-QPS (M-QPS). In the presence of heterogeneity partially synchronized states reminiscent to collective chaos, QPS and M-QPS persist. In addition, the presence of heterogeneity greatly amplifies the differences between the incoherence stability boundaries of excitation and inhibition. Finally, we compare our results with those of a traditional (Wilson Cowan-type) firing rate model with time delays. The oscillatory instabilities of the traditional firing rate model qualitatively agree with our results only for the case of inhibitory coupling with strong heterogeneity.
\end{abstract}

DOI: 10.1103/PhysRevE.98.042214

\section{INTRODUCTION}

Since the seminal work of Hodgkin and Huxley [1], spiking neuron models have been the standard mathematical tool to investigate the collective dynamics of neuronal networks. These models account for the basic properties of neuronssub-threshold voltage dynamics, spiking, and discontinuous synaptic interactions - and hence networks of spiking neurons are considered to be biologically realistic. Yet, network models of spiking neurons are generally not amenable to analysis and hence mostly constitute a computational tool.

Alternatively, researchers use simplified models which describe some measure of the mean activity in a population of cells, customarily taken as the firing rate [2]. Such mean field models (that here we call "traditional firing rate models" or simply "firing rate models") faithfully capture the main types of qualitative dynamical states observed in large populations of asynchronously spiking neurons, and can be mathematically analyzed using standard techniques for differential equations; see, e.g., Refs. [3-6]. Despite their popularity, traditional firing rate models have two major limitations which strongly limit their range of applicability in neuroscience. First, these models are not accurate in describing the dynamics of collective states where a significant fraction of the neurons fires spikes in synchrony. Second, firing rate models do not

*Corresponding author: federico.devalle@upf.edu generally represent proper mathematical reductions of the original network but rather are heuristic. As such there is in general no precise relationship between the parameters in the traditional firing rate model and those in the full network of spiking neurons, and thus there is no clear link between the macroscopic states of the network with the microscopic dynamics of the constituent neurons.

An important example of the application of traditional firing rate models occurs in the analysis of neuronal networks with time delays. It is well-known that synaptic and dendritic processing, as well as axonal propagation, produce unavoidable time delays in the neuronal interactions which profoundly shape the oscillatory dynamics of spiking neuron networks. The study of large networks of spiking neurons with time delays is convoluted, and in this context the mathematical and numerical analysis of firing rate descriptions have been particularly productive; see, e.g., Refs. [7-25]. Yet, how much of the actual dynamics of a large network of spiking neurons with synaptic delays can be captured using traditional firing rate descriptions?

In this paper, we investigate the collective dynamics of a large system of heterogeneous quadratic integrate-and-fire (QIF) neurons with synaptic delays. To perform the analysis we exploit a novel low-dimensional firing rate model that can be exactly derived from the population of QIF neurons [26]. Therefore we use a system of firing rate equations (FRE) that, in contrast with traditional firing rate models, faithfully reproduce all possible collective states of the network. The 
mathematical analysis of the FRE allows us to obtain exact formulas for the boundaries of stability of asynchronous states in both homogeneous and heterogeneous networks. In conjunction with this analysis, we conduct numerical simulations of the corresponding network of QIF neurons to investigate the microscopic states associated with the macroscopic dynamics of the FRE. This combined analysis reveals the presence of large regions of oscillatory states which are unreachable using traditional firing rate models. Some of these states are particularly interesting and we investigate them in detail. They already arise in populations of identical inhibitory neurons, in parameter regions where both the fully synchronous and the asynchronous states are unstable. Hence, in these regimes, the system settles somewhere in between full order and disorder, at a state often called "partial synchrony" [27]. Such partially synchronous states in networks of identical units are self-organized collective states in which the properties of the mean field cannot be trivially inferred from the intrinsic dynamics of the units, but are an emergent property of the network. Here we find three different types of partially synchronous states (with periodic, quasiperiodic, and chaotic mean field dynamics) and also investigate how these states change as neurons are made heterogeneous.

Our work builds primarily on the results by two of the authors [28] about the dynamics of networks of identical, self-oscillatory QIF neurons. Here we extend the results in Ref. [28] in several ways:

(1) The analysis is not restricted to self-oscillating QIF neurons, but extends to networks of excitable QIF neurons.

(2) We perform a detailed numerical exploration of the partially synchronous states and their bifurcations, supported by the systematic computation of the Lyapunov exponents. This allows us to uncover a transition to a novel state which we call modulated quasiperiodic partial synchronization (MQPS), as well as a "quasiperiodic route" to collective chaos. Additionally we investigate how partially synchronous states transform as neurons are made heterogeneous. To the best of our knowledge, this problem has not been addressed in previous work investigating partial synchronization in different populations of identical oscillators [29-41].

(3) We obtain the phase diagram corresponding to populations of heterogeneous QIF neurons. Heterogeneity magnifies the difference between the dynamics of inhibitory and excitatory networks. The phase diagram is finally compared with that of a traditional firing rate model, which we heuristically obtain from the exact FRE obtained in Ref. [26]. The oscillatory instabilities of the two firing rate models qualitatively agree only for the case of inhibitory networks with strong heterogeneity.

The paper is organized as follows. In Sec. II we present the time delayed QIF network model under study. In Sec. III we introduce and discuss the low-dimensional FRE derived, in the large system-size limit, from the QIF network. In Sec. IV we complement the theoretical analysis of the FRE with numerical simulations of the partially synchronous states (QPS, M-QPS, collective chaos). In Sec. V we analyze the effect of heterogeneities in the system dynamics. Finally, in Sec. VI we discuss our results and compare them with those obtained using a traditional firing rate description.

\section{MODEL DESCRIPTION}

We consider a network of $N \gg 1$ all-to-all coupled QIF neurons. The membrane potential of the neurons is governed by the following quadratic differential equation [42]:

$$
\tau \dot{V}_{j}=V_{j}^{2}+I_{j} \quad j=1, \ldots, N
$$

where $\tau$ is a time constant. Every time the membrane potential of a neuron reaches an upper threshold $V_{\text {th }} \gg 1$ it is said to fire. Obviously, in addition to Eq. (1), one must define a spikeresetting condition:

$$
\text { If } V_{j}>V_{\text {th }}, \text { then } V_{\text {reset }} \leftarrow V_{j} \text {. }
$$

In our theoretical analysis we consider the limits $V_{\text {th }}=$ $-V_{\text {reset }} \rightarrow \infty$, which is faithfully reproduced in numerical simulations in the following way: first, we consider $V_{\text {th }}=$ $-V_{\text {reset }}=500$. Then, after the firing, we set the neuron at $V_{\text {reset }}$ after an inactive period of $2 \tau / V_{\mathrm{th}}$. This is the approximate time that a neuron needs to reach $+\infty$ from $V_{\text {th }}$ and return from $-\infty$ to $V_{\text {reset }}$ [43].

The input in Eq. (1) is determined by two distinct contributions:

$$
I_{j}=\eta_{j}+J s(t) .
$$

The first term represents the quenched heterogeneity, which for neurons in the oscillatory regime $\left(\eta_{j}>0\right)$, determines the intrinsic interspike interval (ISI),

$$
T_{j}=\pi \tau / \sqrt{\eta_{j}} .
$$

The second term corresponds to the mean field coupling, where $J$ is the coupling strength and $s(t)$ is the mean synaptic activation. We consider networks of spiking neurons with delayed, mean-field coupling,

$$
s(t)=\frac{\tau}{N \tau_{s}} \sum_{j=1}^{N} \sum_{k} \int_{t-D-\tau_{s}}^{t-D} \delta\left(t^{\prime}-t_{j}^{k}\right) d t^{\prime},
$$

where $t_{j}^{k}$ is the time of the $k$ th spike of neuron $j$, and $\tau_{s}$ the synaptic time constant. After adopting the thermodynamic limit, $N \rightarrow \infty$, we take the limit $\tau_{s} \rightarrow 0$, so that $s$ becomes proportional to the instantaneous population-averaged firing rate at time $t-D$ :

$$
\lim _{\tau_{s} \rightarrow 0} \lim _{N \rightarrow \infty} s(t)=\tau r(t-D) \equiv \tau r_{D}
$$

Finally, we assume a Lorentzian (Cauchy) distribution of the quenched heterogeneity,

$$
g(\eta)=\frac{\Delta / \pi}{(\eta-\bar{\eta})^{2}+\Delta^{2}} .
$$

\section{LOW-DIMENSIONAL DESCRIPTION: FIRING RATE EQUATIONS}

In the thermodynamic limit, the network of QIF neurons can be reduced to a finite set of FRE [26,44]. This is possible assuming that the conditional neuron densities $\rho(V \mid \eta, t)$ are Lorentzian for all $\eta$ values [26], which is mathematically equivalent as to invoke the so-called Ott-Antonsen (OA) theory [45].

Specifically, the original work by Ott and Antonsen applies to the Kuramoto model, and it shows that the model admits 
an exact, low-dimensional description in terms of the $\mathrm{Ku}$ ramoto order parameter [45]. The same theory holds for large populations of globally pulse-coupled oscillators [46], and in particular for ensembles of $\theta$ neurons [47-52]. The $\theta$-neuron phase-model can be transformed to a voltage-based description, the QIF model [53]. Similarly, the macroscopic description for networks of $\theta$ neurons (in terms of the Kuramoto order parameter) transforms into a more natural description for ensembles of QIF neurons in terms of two mean-field quantities of particular relevance in neuroscience: the mean firing rate and the mean membrane potential [26].

Such firing rate description for ensembles of QIF neurons is remarkably simple and amenable to mathematical analysis. This has motivated a number of recent extensions of the FRE for QIF neurons to a number of different setups [54-61]. In particular, considering the QIF model in Sec. II, the FRE consist of a system of two delay differential equations for the firing rate $r$ and for the mean membrane potential,

$$
v=\int_{-\infty}^{\infty} d \eta g(\eta)\left[\lim _{R \rightarrow \infty} \int_{-R}^{R} d V \rho(V \mid \eta, t) V\right],
$$

which read [26,28]

$$
\begin{aligned}
\tau \dot{r} & =\frac{\Delta}{\pi \tau}+2 r v, \\
\tau \dot{v} & =v^{2}+\bar{\eta}-(\pi \tau r)^{2}+J \tau r_{D} .
\end{aligned}
$$

These FRE describe the evolution of the population of infinitely many spiking neurons in terms of the firing rate $r$ and the mean-membrane potential $v$ of the population of QIF neurons Eq. (1). Equations (7) have five parameters, which can be reduced to three by nondimensionalization. In Ref. [28] the FRE Eqs. (7) were analyzed under the restriction $\bar{\eta}>0$, and they were rescaled accordingly. Such rescaling allows to systematically vary the time delay parameter $D$ (including the case $D=0$ ), and facilitates the comparison with the classical and well-studied Kuramoto model with delay [62-66].

Alternatively, here we consider a new nondimensionalization which allows us to investigate the dynamics of the FRE Eqs. (7) in the entire range of $\bar{\eta}$, so that the majority of the neurons can be either self-oscillatory $(\bar{\eta}>0)$ or quiescent/excitable $(\bar{\eta}<0)$. Specifically, we rescale time and $v$ by $D$ and $\tau$ as

$$
\tilde{t}=D^{-1} t, \quad \tilde{v}=D \tau^{-1} v,
$$

so that the new, nondimensional rate is $\tilde{r}=D r$. Then the dynamics of the FRE can be completely explored, without loss of generality, considering the rescaled parameters

$$
\tilde{J}=D \tau^{-1} J, \quad \tilde{\bar{\eta}}=D^{2} \tau^{-2} \bar{\eta}, \quad \tilde{\Delta}=D^{2} \tau^{-2} \Delta,
$$

and setting $\tau=D=1$ in Eqs. (7). Specifically, we investigate the nondimensional system of equations

$$
\begin{aligned}
& \frac{d \tilde{r}}{d \tilde{t}}=\frac{\tilde{\Delta}}{\pi}+2 \tilde{r} \tilde{v}, \\
& \frac{d \tilde{v}}{d \tilde{t}}=\tilde{v}^{2}+\tilde{\bar{\eta}}-(\pi \tilde{r})^{2}+\tilde{J} \tilde{r}_{D=1} .
\end{aligned}
$$

To lighten the notation we drop the tildes hereafter (also in the figure labels).

\section{POPULATIONS OF IDENTICAL NEURONS}

As we discussed previously, the case of identical oscillatory neurons has been investigated in Ref. [28] using a certain rescaling that required $\bar{\eta}>0$. Here we adopt the rescaling in Eq. (8), which allows us for an exhaustive investigation of the dynamics of the system by systematically varying the parameter $\bar{\eta}$.

Before starting the analysis, we emphasize that the Lorentzian ansatz (or the equivalent OA ansatz) is not strictly valid for identical oscillators. In this case the system is partially integrable and its phase space is foliated by a continuum of invariant manifolds, being the Lorentzian ansatz a particular one. Actually, for the case of identical neurons $(\Delta=0)$, the correct approach is to resort to the so-called WatanabeStrogatz theory [67], instead of the OA ansatz [60,68]. Nevertheless, from a physical perspective the OA/Lorentzian ansatz is very significant since any small amount of noise and/or heterogeneity destroys the degeneracy and, at least for the systems analyzed so far, the density converges to a vicinity of the OA manifold [69].

Hence, in the following we analyze the identical case taking into account that its full significance holds once a small amount of noise or heterogeneity is added to the system. However, to avoid the inclusion of noise/heterogeneity in the integration algorithm, we use initial conditions in the Lorentzian manifold in all the numerical simulations of ensembles of identical QIF neurons Eqs. (1).

\section{A. Analytical results: The incoherent and the fully synchronized states}

\section{The incoherent state}

Equation (9) has at most four fixed points. In some parameter values one of these points is located in the negative rate region $(r<0)$, and we refer to it as "unphysical." Moreover, for $\Delta=0$, the axis $r=0$ is invariant so that solutions initiated with $r(0)>0$ remain positive for all times. The equilibria of Eqs. (9) can be grouped into two sets of fixed points:

(1) The first pair of fixed points is located in the $(r, v)$ plane at

$$
\mathbf{a}_{ \pm}=\left(\frac{J \pm \sqrt{J^{2}+4 \pi^{2} \bar{\eta}}}{2 \pi^{2}}, 0\right) .
$$

For $J>0$, these fixed points are born at a saddle-node bifurcation located at

$$
J_{\mathrm{sn}}=2 \pi \sqrt{-\bar{\eta}}
$$

This line is partly depicted as a solid green straight line in the phase diagram Fig. 1, and is located in the region $\bar{\eta}<0$. Note that the fixed point $\mathbf{a}_{-}$becomes unphysical for $\bar{\eta}>0$, while $\mathbf{a}_{+}$exists for $J<0$ only if $\bar{\eta}>0$. As shown below, the fixed point $\mathbf{a}_{+}$is stable in a wide range of parameter values. We will refer to $\mathbf{a}_{+}$as the incoherent, or the asynchronous state. For finite networks $\mathbf{a}_{+}$becomes a so-called splay state, with all neurons firing with the same ISI, and one neuron firing every ISI/ $N$ time units.

(2) The second pair of fixed points,

$$
\mathbf{q}_{ \pm}=(0, \pm \sqrt{-\bar{\eta}})
$$




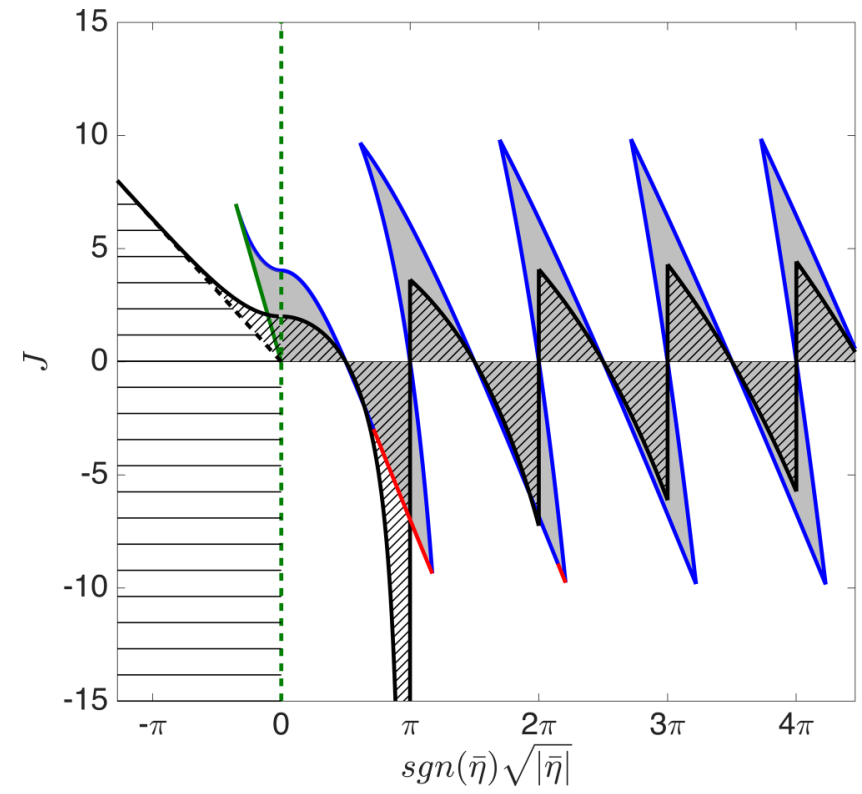

FIG. 1. Phase diagram for identical neurons, $\Delta=0$. Shaded region: The asynchronous state $\left(\mathbf{a}_{+}\right)$is stable. Slantwise hatched region: full synchrony is unstable. Horizontally hatched region: The fully synchronized state does not exist and the only attractor is the global rest state $\mathbf{q}_{-}$. The orbit of fully synchronized self-sustained oscillations is created at the dashed black line (at $\bar{\eta}<0$ ), Eq. (14). Blue and red lines are the loci of the sub- and super-critical Hopf-like instabilities of incoherence Eqs. (10). Solid green line: saddle-node bifurcation. The vertical dashed green line separates the oscillatory from the excitable regime of the QIF neuron.

only exists for $\bar{\eta}<0$. They correspond to quiescent states, and coincide with the fixed points of an individual QIF neuron. Hence, $\mathbf{q}_{-}$(respectively, $\mathbf{q}_{+}$) is trivially stable (unstable). The bifurcation at $\bar{\eta}=0$ (green dashed line in Fig. 1) is somewhat peculiar because it is not a simple saddle-node bifurcation of $\mathbf{q}_{+}$and $\mathbf{q}_{-}$as expected. For $J>0$, it involves the simultaneous collision with $\mathbf{a}_{-}$, while for $J<0$ it coincides with the appearance of $\mathbf{a}_{+}$for $\bar{\eta}>0$.

Next we study the linear stability of the fixed points. The incoherent state $\mathbf{a}_{-}$is always unstable, while the linear stability analysis of the high activity, asynchronous state $\mathbf{a}_{+}$reveals interesting features. Imposing the condition of marginal stability $\lambda=i \Omega$ in the linearization of Eq. (9), we find a family of oscillatory instabilities at

$$
J_{H}^{(n)}=\pi\left(\Omega_{n}^{2}-4 \bar{\eta}\right) \times\left\{\begin{array}{l}
\left(6 \Omega_{n}^{2}+12 \bar{\eta}\right)^{-1 / 2}, \text { odd } n \\
\left(2 \Omega_{n}^{2}-4 \bar{\eta}\right)^{-1 / 2}, \text { even } n
\end{array}\right.
$$

where $\Omega_{n}=n \pi$. We point out that these instabilities (represented as blue and red lines in the phase diagram Fig. 1) are actually Hopf-like, rather than Hopf, because of two facts: (i) The amplitude equations, computed in the Supplemental Material of [28], are degenerated. (ii) In the supercritical case, we find that the emerging limit cycle has a period $2 \pi / \Omega_{n}$, which remains constant as one moves away from threshold. This is apparently related to the reversible character of Eqs. (9) for $\tilde{\Delta}=0$ (note the invariance $t \rightarrow-t, v \rightarrow-v$ ) that, as argued in Ref. [28], stabilizes symmetric orbits with fixed periods when $D$ is nonzero.

\section{The fully synchronized state}

Besides the stability boundary of the asynchronous state, we can also analytically determine the boundaries of full synchrony, $V_{j}=V(t), \forall j$. The FRE Eq. (9) are not suitable for this analysis, since the fully synchronized state corresponds to a degenerate infinite trajectory along the $v$ axis. Full synchrony is hence investigated using the original Eqs. (1).

As shown in Ref. [28], for oscillatory dynamics $(\bar{\eta}>0)$ the stability region of full synchrony is bounded by the family of curves

$$
J_{s}^{\left(n^{\prime}\right)}=2 \sqrt{\bar{\eta}} \cot \left(\frac{\sqrt{\bar{\eta}}}{n^{\prime}}\right) \quad \text { with } \quad n^{\prime}=1,3, \ldots,
$$

and by the evenly spaced lines $\sqrt{\bar{\eta}}=m \pi$ with $m=1,2,3, \ldots$.

However, in the case $\bar{\eta}<0$, we emphasize that the term "full synchronization" cannot be strictly used since the neurons are excitable and not self-sustained oscillators. However, to simplify the notation, in the following we refer to collective oscillatory states with $\bar{\eta}<0$ as fully synchronized states. Indeed, due to the presence of time delay, collective selfsustained oscillations could be in principle maintained for strong enough excitatory coupling. To study the existence and stability of these states, we rewrite the QIF model Eq. (1) as

$$
\dot{V}_{j}=V_{j}^{2}-|\bar{\eta}|+J r_{D}
$$

Then, to investigate the existence of a fully synchronized state, we can drop the index $j$ in Eq. (12). Note that, in the absence of coupling, Eq. (12) has one stable $(s)$ and one unstable $(u)$ fixed points

$$
V_{u}^{*}=-V_{s}^{*}=\sqrt{|\eta|} .
$$

Between consecutive spikes, the evolution of the membrane potential of all neurons is given by

$$
\dot{V}=V^{2}-|\bar{\eta}| \text {. }
$$

Considering that the neurons' membrane potentials reach infinity at $t=0$, we find that their membrane potentials at the time immediately before receiving the spike, $t=D^{-}=1^{-}$, must satisfy the following equation:

$$
\int_{-\infty}^{V\left(1^{-}\right)} \frac{d V}{V^{2}-|\bar{\eta}|}=1
$$

which gives

$$
V\left(1^{-}\right) \equiv V^{-}=-\sqrt{|\bar{\eta}|} \operatorname{coth} \sqrt{|\bar{\eta}|}
$$

A necessary condition for the existence of self-sustained collective oscillations is that an excitatory spike causes a jump in $V$ beyond the unstable fixed point, which enables the repetition of the cycle. More precisely, immediately after receiving the first spike, $t=1^{+}$, the membrane potential $V^{+}$ must satisfy $V^{+}>V_{u}^{*}$. Then, for $\bar{\eta}<0$, we find that fully synchronized solutions exist above the critical coupling,

$$
J_{c}=V_{u}^{*}-V^{-}=2 \sqrt{|\bar{\eta}|} \frac{e^{2 \sqrt{|\bar{\eta}|}}}{e^{2 \sqrt{|\bar{\eta}|}}-1} .
$$


To analyze the stability of full synchrony, we study the evolution of an infinitesimal perturbation $\delta V$ of a single neuron membrane potential away from the cluster formed by the rest of the population. The perturbed neuron and the cluster before the incoming spike evolve according to the flow given by Eq. (13). The multiplier of the linearized flow $(\delta \dot{V}=$ $2 V \delta V$ ) is antisymmetric causing convergence for negative $V$, and divergence for positive $V$. Hence, to have a stable fully synchronous solution, the neurons need to spend more time in the convergent region of the flow than in the divergent one. This holds if the instantaneous jump of the membrane potential due to the incoming spike is large enough. Then the critical coupling corresponds to $V^{+}=\left|V^{-}\right|$, i.e., $J_{s}=2\left|V^{-}\right|$, or

$$
J_{s}=2 \sqrt{|\bar{\eta}|} \operatorname{coth} \sqrt{|\bar{\eta}|} .
$$

This function is precisely the boundary in Eq. (11) with $n^{\prime}=$ 1 , which extends to the negative $\bar{\eta}$ region, since $\cot (i x)=$ $-i \operatorname{coth}(x)$. Note also that $J_{s}$ approaches $J_{c}$ as $\bar{\eta} \rightarrow-\infty$.

\section{B. Phase diagram}

The phase diagram shown in Fig. 1 summarizes our analytical results for populations of identical neurons. On the $y$ axis we represent the coupling strength $J$, which can be either excitatory or inhibitory. On the $x$ axis we represent a quantity that, if positive, is proportional to the natural frequency of the neurons; see Eq. (4). Regions with qualitatively different dynamics are highlighted with different combinations of colors and patterns. In the gray shaded regions, the asynchronous state $\mathbf{a}_{+}$is stable, while slantwise hatching indicates instability of the fully synchronized state. On the other hand, in the horizontally hatched area, the global quiescent state $\mathbf{q}_{-}$ is the only attractor of the system. In the unhatched white region, full synchrony is a stable attractor (and typically the only one-see below), but several of such states may coexist in certain regions for $\bar{\eta}>0$.

More specifically, in the excitable region $(\bar{\eta}<0)$ of the diagram the global quiescent state $\mathbf{q}_{-}$is always stable. In addition, the stability region of the asynchronous state $\mathbf{a}_{+}$(gray shading) is bounded by the saddle-node bifurcation $J_{\mathrm{sn}}$ (green line), and the Hopf-like bifurcation line $J_{H}^{(1)}$, Eq. (10) (blue line). The two lines meet at a Zero-Hopf codimension-two point. In the unhatched gray region $\mathbf{a}_{+}$coexists not only with q- but also with the fully synchronized state. This oscillatory state becomes stable at the solid black line Eq. (15).

On the other hand, the positive $\bar{\eta}$ region of the diagram is characterized by a sequence of subcritical (blue lines) and supercritical (red lines) Hopf-like bifurcations, defined by Eq. (10), that switch the stability of the incoherent state $\mathbf{a}_{+}$. Remarkably, in this region (where neurons are self-sustained oscillators), the phase diagram bears strong resemblance with that of the Kuramoto model with time delays [62-66]. The two systems display tent-shaped regions with an even spacing given by the equality between the delay $(D=1)$ and the intrinsic ISI Eq. (4), as well as bistability regions between full sync and incoherence (unhatched gray regions). However, while in the Kuramoto model the Hopf bifurcations are always subcritical, here we find supercritical Hopf bifurcations for some $\bar{\eta}$ values in the inhibitory $(J<0)$ part of the diagram.
Near the supercritical Hopf bifurcations, in the unshaded hatched region, both the incoherent and the fully synchronous states are unstable, and partial synchrony (QPS, M-QPS, and collective chaos) is found. In the next section we classify these states in terms of their macroscopic and microscopic dynamics, and investigate their bifurcations.

Finally, we discuss an interesting feature of the phase diagram in Fig. 1-see also the phase diagram in Ref. [28]. Note that, at variance with the vertically oriented, tent-shaped regions of the Kuramoto model [62-66], here the regions of stability are tilted. This discrepancy between populations of QIF neurons and the Kuramoto model can be understood as follows: in the QIF model the neurons always advance their phase in response to excitatory inputs, and always delay their phase in response to inhibitory inputs-i.e., they have a so-called Type 1 phase resetting curve. This produces the progressive "advancement" of the boundaries in the excitatory part of the phase diagram as the strength of the excitatory coupling $J$ is increased-given that neurons increase their firing frequency and thus their effective value of $\bar{\eta}$. Similarly, in the inhibitory region, the neurons slow down their firing frequency in response to inhibitory inputs, and this progressively "delays" the boundaries for $J<0$. In contrast, in the classical Kuramoto model, the terms producing advances and delays in response to excitation and inhibition are not included [70], and hence the boundaries are not tilted.

\section{Numerical analysis of partially synchronous states}

Next we perform a numerical exploration of the partially synchronized states arising both in the white slantwisehatched region of Fig. 1, as well as in some neighboring regions. In Table I these partially synchronized states are classified according to their dynamics, both for identical and for heterogeneous (in Sec. V) populations of QIF neurons. The macroscopic dynamics of the states is investigated performing numerical simulations of the FRE Eq. (9), and illustrated in the columns 1 and 2 of Fig. 2. To investigate the single neuron dynamics associated to the macroscopic states we also performed numerical simulations of the original system of QIF neurons Eqs. (1), and depicted the raster plots (column 3), and the ISI return maps (column 4) and histograms (column 5). Finally, in column 1, we also show the time series of the population-mean firing rate of the network simulations (dashed red lines), which show a perfect agreement with the time series of the FRE (blue lines) - except in panel (d1), where the collective dynamics is chaotic.

Note that stable partially synchronized states are not only found in the slantwise-hatched region of Fig. 1, but also in a neighborhood of this region with $\sqrt{\bar{\eta}}>\pi$. This is because the region where the Hopf-like bifurcation $J_{H}^{(1)}$ is supercritical (around the red line at $\sqrt{\bar{\eta}} \approx \pi$ in Fig. 1) extends to $\sqrt{\bar{\eta}}>\pi$, and hence one expects a low-amplitude periodic solution bifurcating from incoherence, $\mathbf{a}_{+}$, coexisting with a fully in-phase synchronized state. In Figs. 2(a1) and 2(a2) we, respectively, show the time series and the phase portraits corresponding to the numerical integration of Eqs. (9) for $\sqrt{\bar{\eta}}=3.6$. These simulations confirm the presence of a small amplitude symmetric limit cycle, which grows in size as parameters are moved away from the instability. 
TABLE I. Classification of the different dynamical states observed for populations of both identical, and heterogeneous QIF neurons. The names of the states are the following: ASYNC: Asynchronous or incoherent state. FULL SYNC: fully synchronized state. QPS: Quasiperiodic partial synchronization. M -QPS: modulated quasiperiodic partial synchronization. PS-I and PS-II stands for type I and type II partially synchronous states. M-PS: modulated partially synchronous state. The prefix $2 \mathrm{~F}$ - and $3 \mathrm{~F}$ - indicate the number of frequencies of the corresponding quasiperiodic dynamics. For each state we specify the dynamics at the macroscopic level (mean field) and at the microscopic level (single neuron). For the states of collective chaos, $\lambda$ is the Lyapunov exponent of a single neuron forced by the mean field.

\begin{tabular}{lcccccc}
\hline \hline & & ASYNC & FULL SYNC \\
PDENTICAL & $\begin{array}{c}\text { Single neuron: } \\
\text { Mean field: }\end{array}$ & $\begin{array}{c}\text { Periodic } \\
\text { Constant }\end{array}$ & $\begin{array}{c}\text { QPS } \\
\text { Periodic }\end{array}$ & $\begin{array}{c}\text { MF-Quasip. } \\
\text { Periodic }\end{array}$ & $\begin{array}{c}\text { 3F-Quasip. } \\
\text { 2F-Quasip. }\end{array}$ & $\begin{array}{c}\text { COLECTIVE CHAOS } \\
\text { Chaotic-like }(\lambda=0) \\
\text { Chaotic }\end{array}$ \\
\hline & & ASYNC & PS-I & PS-II & M-PS & COLLECTIVE CHAOS \\
HETEROGENEOUS & Single neuron: & Periodic & $\begin{array}{c}\text { Periodic, } \\
\text { Periodic, }\end{array}$ & $\begin{array}{c}\text { 2F-Quasip., } \\
\text { 2F-Quasip. }\end{array}$ & $\begin{array}{c}\text { 2F-Quasip. } \\
\text { 3F-Quasip. }\end{array}$ & $\begin{array}{c}\text { Chaotic-like }(\lambda<0) \\
\text { Chaotic }\end{array}$ \\
& Mean field: & Constant & Periodic & Periodic & 2F-Quasip. & \\
\hline \hline
\end{tabular}

As analyzed in Ref. [28], in Fig. 2(a1) the oscillation period of the mean field is exactly $T=2$ (or, in dimensional form, $T=2 D$ ). The periodic dynamics of the global quantities leads to quasiperiodic dynamics of the individual neurons, i.e., Quasiperiodic partial synchrony (QPS). This may be appreciated plotting the ISIs of a single neuron versus their consecutive ISIs. The resulting return plot, shown in Fig. 2(a4), forms a closed curve indicating quasiperiodic dynamics. Interestingly, the ISIs of the neurons are always shorter than the period of the firing rate oscillations, as shown by the ISI histogram in Fig. 2(a5). The bimodal structure of the distribution is related to double-loop shape of the macroscopic periodic attractor.

The limit cycle that emerges via the Hopf-like instability displays a robust $v \rightarrow-v$ symmetry that only breaks down after another bifurcation. In Ref. [28], for $\sqrt{\bar{\eta}}=3$, it was shown that symmetry broke down after a period-doubling bifurcation. Here, taking a slightly larger value of $\sqrt{\bar{\eta}}$ and increasing inhibition, we observe an imperfect symmetry breaking transition, with two coexisting attractors; see Figs. 2(b1),

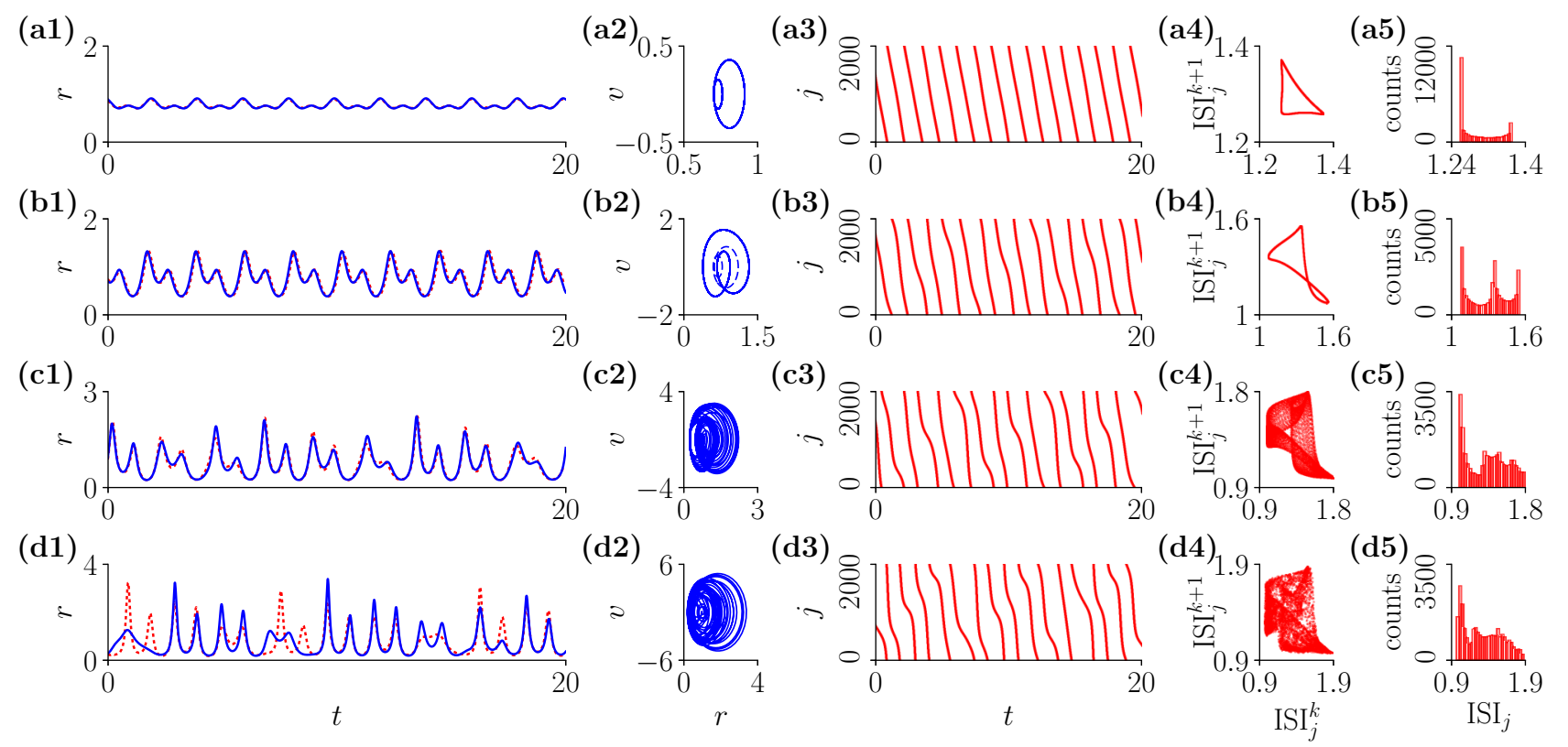

FIG. 2. Macroscopic (columns 1 and 2) and microscopic (columns 3-5) dynamics of QPS (rows a and b), M-QPS (row c) and collective chaos (row d), see Table I. Column 1: time series of the mean firing rate. Blue lines correspond to numerical simulations of the FRE Eqs. (9), while red dotted lines are obtained computing the mean firing rate of a population of $N=2000$ QIF neurons. Column 2: $(r, v)$ phase portraits, numerically obtained using Eqs. (9). In panel (b2) two coexisting periodic attractors are shown: QPS-asym(I) (solid) and QPS-asym(II) (dashed)—see also inset of Fig. 3. Panels (b1, b3-b5) correspond to QPS-asym(I). Columns (3-5) show the dynamics of a population of $N=2000$ QIF neurons. Column 3: raster plots. Neurons are ordered according to their firing time at the beginning of the simulation (due to the first order nature of the QIF model, this ordering is preserved in time). Columns 4 and 5 show return ISI plots and ISI distributions of an arbitrary neuron $j$. The return plots of panels (a4, b4) are closed curves, indicating quasiperiodic microscopic dynamics in the QPS-sym and QPS-asym. The corresponding ISI histograms (a5, b5) show two (QPS-sym) or three (QPS-asym) peaks. In the M-QPS, neurons are quasiperiodic with three characteristic frequencies - the return plots of panel (c4) is a closed surface in 3D, and therefore its projection in 2D fills a defined region of the space. Parameters: (row a) $J=-9.2$ (row b) $J=-9.5$, (row c) $J=-10.3$, (row d) $J=-10.6$. We use $\sqrt{\bar{\eta}}=3.6$ in all simulations. 

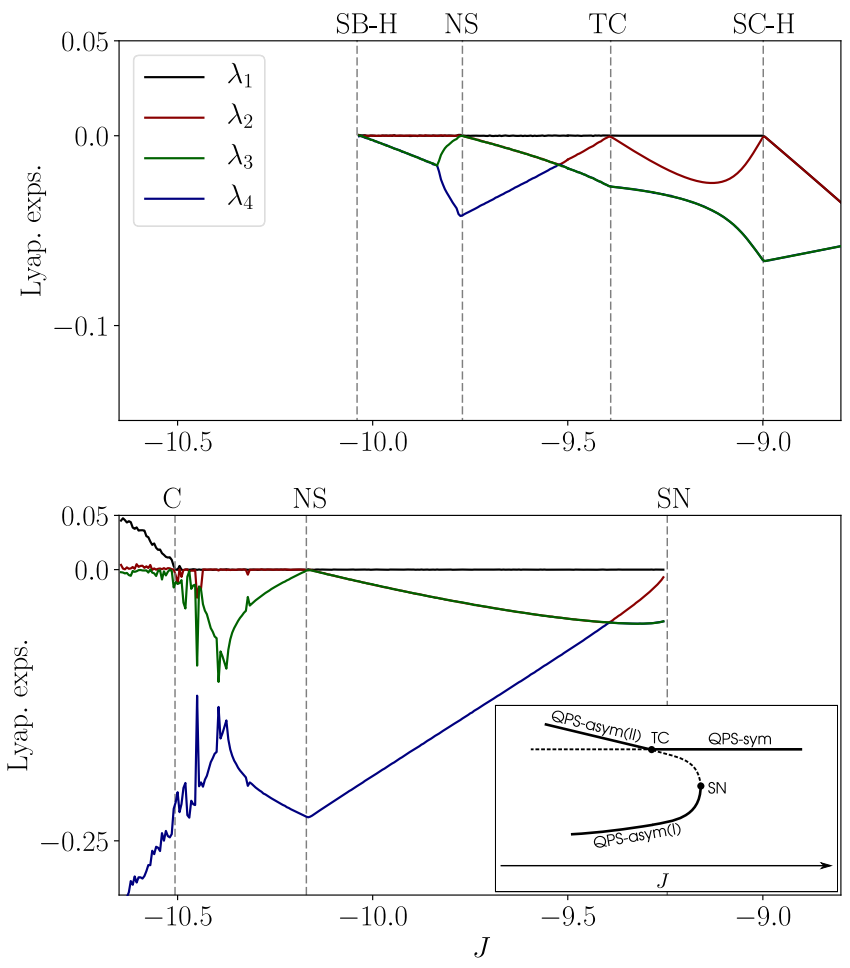

FIG. 3. Four largest Lyapunov exponents for two alternative bifurcation sequences in a range of negative $J$ values and fixed $\sqrt{\bar{\eta}}=3.6$. For each solution, the continuation was carried out either increasing or decreasing parameter $J$ adiabatically. In the top panel the vertical dashed lines indicate, from right to left: a supercritical Hopf bifurcation (SC-H), a transcritical bifurcation (TC), a NeimarkSacker bifurcation (NS), and a subcritical Hopf bifurcation (SB-H). In the bottom panel the vertical dashed lines indicate, from right to left: a saddle-node bifurcation (SN), a Neimark-Sacker bifurcation (NS), and the onset of chaos (C). The inset shows a sketch of the bifurcation diagram connecting the two bifurcation sequences.

2(b2), and 3. These asymmetric periodic orbits-which we call QPS-asym(I) and QPS-asym(II) - are not related by symmetry. In fact, each attractor is born via a different bifurcation, see details below. In these asymmetric states the period differs from $2 D$, but still neurons are quasiperiodic, see Figs. 2(b4) and 2(b5).

Increasing inhibition further, the macroscopic dynamics becomes more irregular, with no evident periodicity; see Figs. 2(c1) and 2(c2). Below, we show the analysis of the Lyapunov exponents indicating quasiperiodic mean field dynamics with two incommensurable frequencies. As a consequence of this quasiperiodic forcing, the neurons exhibit three-frequency quasiperiodic motion; see Fig. 2(c4). We call this new state modulated QPS, or simply M-QPS, due to the additional modulating frequency. To the best of our knowledge this state has been only reported in a very different setup [29,71]. Lowering $J$ further, the M-QPS eventually turns into a chaotic state; see Figs. 2(d1) and 2(d2).

To determine the bifurcations linking different partially synchronous states (QPS, M-QPS, or collective chaos), we computed the four largest Lyapunov exponents of the FRE on the line along the $J$ direction with $\bar{\eta}$ value of Fig. 2. Employing the usual method [72], parameter $J$ was quasiadiabatically
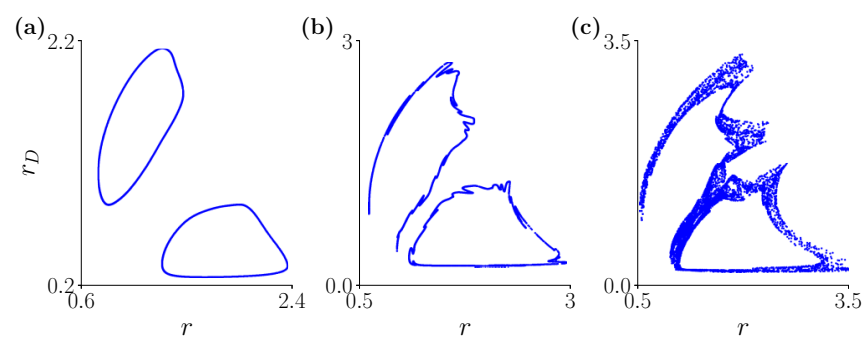

FIG. 4. Poincaré sections of the FRE (9) for $\sqrt{\bar{\eta}}=3.6$, and for three different values of the inhibitory coupling strength: (a) $J=$ -10.3 ; (b) $J=-10.48$; (c) $J=-10.6$. The Poincaré surface is $v=$ $0, \dot{v}<0$.

decreased and increased, to detect eventual bistabilities. Two parallel sequences of bifurcations were eventually detected, as shown in the top and bottom panels of Fig. 3. In the top panel, moving leftwards, the fixed point attractor $\left(\mathbf{a}_{+}\right)$ first undergoes a supercritical Hopf-like bifurcation, after which the stable attractor of the system is a symmetric QPS attractor. The symmetry breaking takes place at a transcritical bifurcation (TC), after which the limit cycle is asymmetric [QPS-asym(II)]. At a lower $J$ value, the asymmetric periodic orbit undergoes Neimark-Sacker bifurcation giving rise to $\mathrm{M}$ QPS_-given that we find two vanishing Lyapunov exponents. Further decreasing inhibition, the M-QPS disappears in a subcritical Hopf bifurcation (SB-H).

In the other sequence of bifurcations-bottom panel of Fig. 3-another asymmetric orbit [QPS-asym(I)] is born at a saddle-node (SN) bifurcation. As QPS-asym(II), it also undergoes a Neimark-Sacker bifurcation as $J$ is decreased giving rise to M-QPS. In Figs. 2 and 4 we show the M-QPS state corresponding to this particular sequence of bifurcations. However, note that M-QPS states resulting from either route in Fig. 3 have the same dynamical features (two vanishing largest Lyapunov exponents and three-frequency microscopic motion). Lockings occur at certain windows of $J$, where the second largest Lyapunov exponent is not zero. To further prove the macroscopic quasiperiodic nature of the M-QPS, we also show Poincaré sections for three different values of $J$ in Fig. 4. As $J$ is lowered the torus corrugates as typically observed in the transition to chaos via fractalization of the torus [73]; see Fig. 4(b). The torus breaks down around $J=-10.5$, and the attractor turns fractal. Notably, the chaotic attractor achieves rapidly an information dimension larger than three according to the Kaplan-Yorke formula [74] since $\lambda_{1}>\left|\lambda_{3}\right|$, see bottom panel of Fig. 3; in contrast with the dimension slightly above two found in Ref. [28] for the chaotic attractor born from the period doubling cascade. It is important to stress that, in spite of the positive Lyapunov exponent (of the collective dynamics), the microscopic dynamics remains nonchaotic, because the individual oscillators have only one degree of freedom. In fact the structure of the model imposes the neurons to fire sequentially; see Fig. 2(d3). Finally, the inset in Fig. 3 is our conjecture of how the two bifurcation sequences in the main panels are connected: the unstable branch the SN bifurcation collides with the symmetric QPS state at the TC bifurcation.

In the preceding figures we have shown the transitions along a specific $\bar{\eta}$ value. Seeking a more global picture we 


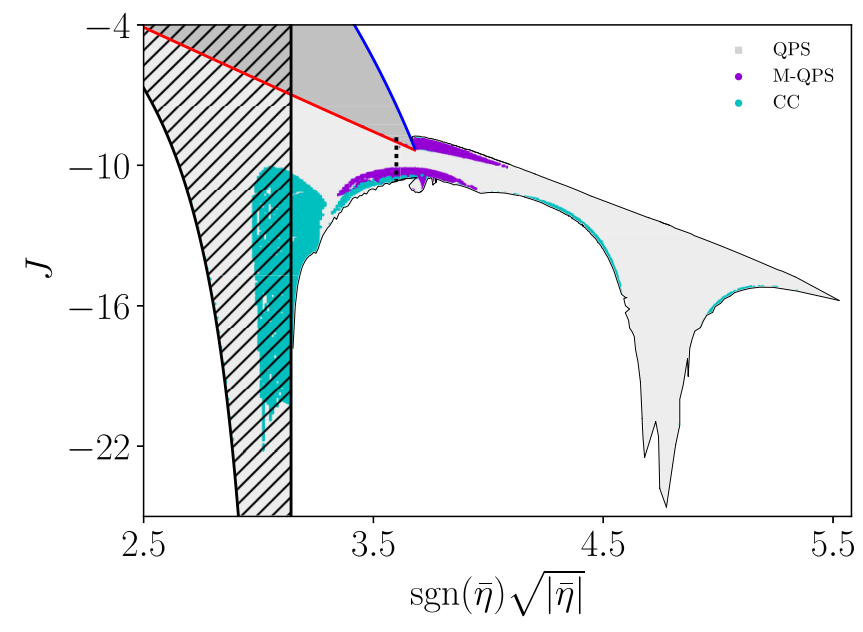

FIG. 5. Numerical exploration of the partially synchronized states (QPS,M-QPS, collective chaos) near the supercritical Hopf bifurcation in phase diagram Fig. 1. In the light gray region the largest Lyapunov exponent is zero, and QPS is stable. The purple dots correspond to two vanishing Lyapunov exponents, indicating quasiperiodic dynamics. In the cyan region the dynamics is chaotic. The vertical dashed black line at $\sqrt{\bar{\eta}}=3.6$ corresponds to the range of parameters explored in Fig. 3.

decided to sweep parameters $J$ and $\bar{\eta}$ monitoring the largest Lyapunov exponents. This permits to identify the attractor types efficiently. Figure 5 shows the region spanned by partially synchronized dynamics [75]. The light gray and purple regions indicate QPS and M-QPS states, respectively, while cyan dots correspond to chaotic dynamics. It surprised us the extension of the parameter region where QPS coexists with full synchrony (light shaded unhatched area). There is a "tongue" extending to very negative $J$ values around $\sqrt{\bar{\eta}}=4.7$ that looks like an "echo" at $3 \pi / 2=4.712 \ldots$ of the infinite tongue just below $\sqrt{\bar{\eta}}=\pi$. We have not an intuitive explanation for this. Quasiperiodic dynamics (M-QPS) is found always not far from the degenerate point were the instability boundaries for $n=1$ and $n=2$, see Eq. (10), meet. This is probably not casual (further analysis is nonetheless beyond our scope [76]). Regarding the chaotic state, it shows up in two distinct regions: the leftmost one is related to the period-doubling scenario observed in Ref. [28], while the rightmost one is correspond to the quasiperiodic route uncovered here.

\section{POPULATIONS OF HETEROGENEOUS NEURONS}

In this section we consider that the neurons in the network are nonidentical, and we investigate how this alters the phase diagram in Fig. 1 and the partially synchronous states depicted in Fig. 2. Hence, in the following we assume that the half-width $\Delta$ of the Lorentzian distribution Eq. (6) is not zero. Under the presence of Lorentzian heterogeneity fully and partially synchronous states discussed previously are unattainable. In the following the generic term "partial synchronization" refers to any state of the network which is not an incoherent state.
States reminiscent of QPS and collective chaos persist for finite values of $\Delta$, with individual neurons displaying different motions depending on their native $T_{j}$ values. We denote these states as partial synchronization-I (PS-I) and PS-II for the states reminiscent of full synchrony and QPS, respectively. In PS-I most neurons are 1:1 entrained to the global frequency, and the remaining neurons are either entrained with a different ratio or display quasiperiodic dynamics. In the case of PS-II only a minority of the neurons entrains $1: 1$ with the macroscopic oscillation. We use the distinction between PS-I and PS-II for convenience, but we emphasize that there is not a clearcut distinction between both states and one can transit from one to the other continuously. As for the other states, the asynchronous state continues to exist after introducing the heterogeneity, although not in the form of a splay state. Finally, M-QPS is replaced by a modulated PS states, or M-PS, while collective chaos continues to exist; see Table I.

Next we analyze how the stability regions of incoherence, which can still be analytically computed from the FRE Eqs. (9), change due to the presence of heterogeneity. Unfortunately, in the heterogeneous case, a stability analysis similar to that of Sec. IV A for the case of synchronous states is not possible. Later in this section we examine how the partially synchronized states found in the region $\bar{\eta}>0$ for identical inhibitory neurons are altered by quenched disorder.

\section{A. Stability boundaries of incoherence and phase diagram for $\Delta=0.1$}

It is important to note that the presence of heterogeneity removes all degeneracies of the FRE Eqs. (9). The fixed points can be still obtained in parametric form, as well as the boundaries corresponding to saddle-node bifurcations of the asynchronous/incoherent states (green lines in Fig. 6). However, these expressions are lengthy and here we omit them for the sake of clarity; see Ref. [26]. Linearizing and imposing the condition for marginal stability, also the loci of the Hopf bifurcations can be obtained in parametric form (black lines in Fig. 6). We finally used numerical simulations of Eqs. (9) to detect the regions where partially synchronous states become unstable, or cease to exist (dark gray region in Fig. 6).

The phase diagram in Figure 6 summarizes these results for $\Delta=0.1$, and displays the regions where distinct dynamics occur-compare with the phase diagram Fig. 1. As expected, close to the $J=0$ axis incoherence is the only attractor of the system (dark shaded). Like in the case of identical neurons, bistability regions between incoherence and another state(s) exist (light shaded). Interestingly, for inhibitory coupling, the Hopf bifurcations of the asynchronous state largely overlap with the numerical boundaries of "pure" incoherence (dark shading). This indicates that, for inhibitory networks, the intervals where the Hopf bifurcations are supercritical are dramatically enlarged as heterogeneity is increased.

\section{Phase diagram in the region $\bar{\eta}<0$}

Figure 7 displays an enlarged view of the phase diagram Fig. 6 , around the brown region located at $\bar{\eta}<0$. The scenario of bifurcations is quite intricate in this region, and here we describe it in detail. The brown shaded region is interesting 


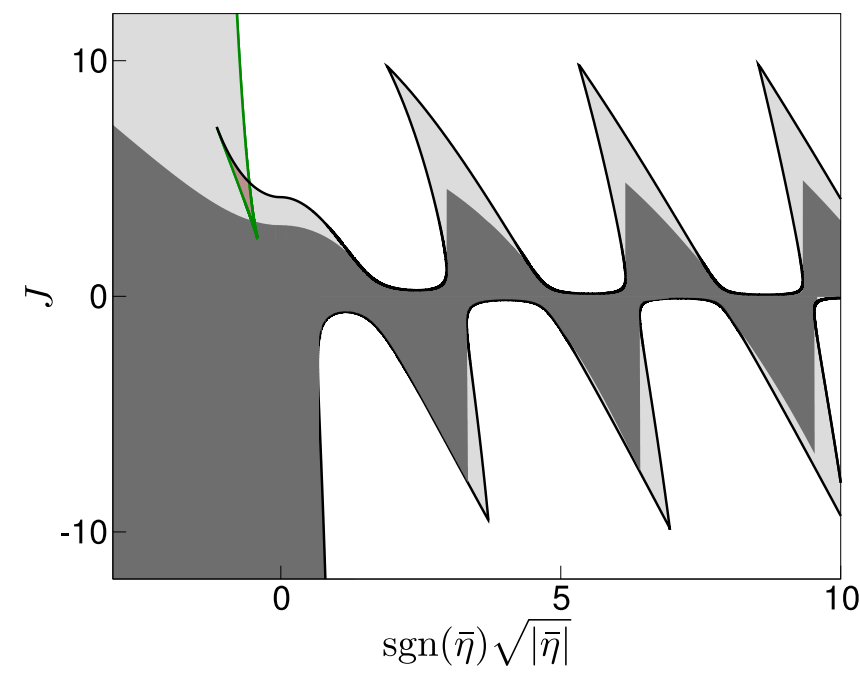

FIG. 6. Phase diagram for populations of heterogeneous neurons, $\Delta=0.1$. Dark shaded region: Incoherence (fixed point) is the only stable state. Light shaded region: Incoherence (fixed point) coexist with a partially synchronous state (limit cycle). Brown region: Two forms of asynchrony (high and a low activity fixed points) coexist with a partially synchronous state. Green lines are saddle-node bifurcations, and black lines correspond to Hopf boundaries. Note that here, in contrast with Fig. 1, the Hopf boundaries are not represented in Blue/Red (we do not explicitly specify whether these boundaries are subcritical or supercritical). The boundary between light and dark shaded regions was obtained numerically.

since a high-rate and a low-rate fixed points - reminiscent of the fixed points $\mathbf{a}_{+}$and $\mathbf{q}_{-}$- coexist with a periodic orbit. In Fig. 7 we have included two dashed lines corresponding to bifurcations involving saddles and/or repellors to fully clarify the transitions between different stable states. We also highlight two codimension-two points: the cusp point where the two saddle-node bifurcations meet, and the zero-Hopf
(ZH) point - associated to a zero and a pair of pure imaginary eigenvalues. The different shadings in the figure indicate regions with qualitatively different attractors: in the dark region (I) only one fixed point is stable. In the small dark purple region (II) this fixed point coexists with another stable fixed point. In the light shaded areas (IV,V,VI) a stable fixed point coexists with a stable limit cycle. This limit cycle is the only stable attractor in the white region (VII). Finally, in the brown region (III), there are three coexisting stable attractors: two fixed points, and a limit cycle.

The transitions between any two regions in the diagram can be understood considering a three-dimensional space. In the right panels of Fig. 7 we present sketches of the phase portraits of the different stability regions, by means of Poincaré sections. Thick lines represent two-dimensional manifolds. Comparing the phase diagram in Fig. 7 with the results previously obtained for instantaneous interactions [26], we see that the delay promotes the appearance of a Hopf bifurcation of the asynchronous state. Note that the scenario shown in Fig. 7 resembles that of a population of heterogeneous QIF neurons with fast synaptic kinetics [54], but here we find a codimension-two $\mathrm{ZH}$ point, instead of a double-zero eigenvalue point.

\section{B. Numerical analysis of partially synchronized states in the presence of heterogeneity}

Here we explore numerically how the presence of heterogeneity transforms the partially synchronized states described in Sec. IV. To circumvent sample-to-sample fluctuations, $\eta_{j}$ values are selected deterministically from the Lorentzian distribution setting $\eta_{j}=\bar{\eta}+\Delta \tan [\pi(2 j-N-1) /(2 N+2)]$, where $j=1,2, \ldots, N$. States reminiscent of previous partially synchronous states persist for $\Delta \neq 0$; in columns ( 1 and 2) of Fig. 8 we show the macroscopic time series of PS-II, MPS and collective chaos, where blue lines represent numerical integration of the FRE Eqs. (9) and red lines simulation of

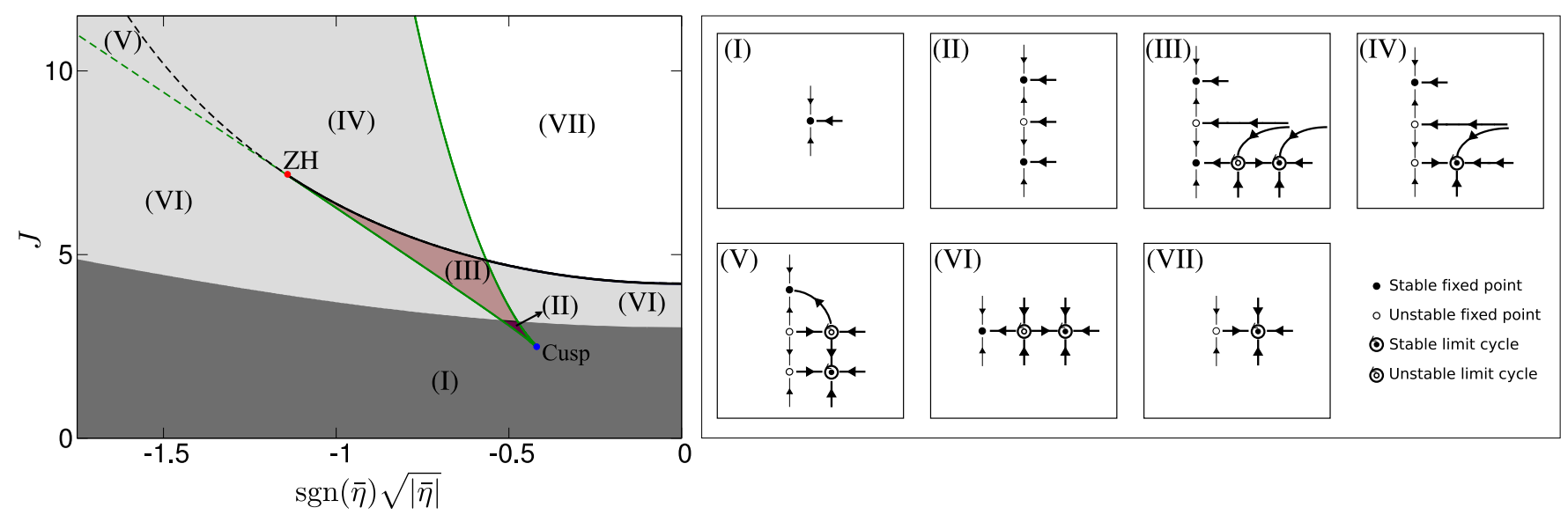

FIG. 7. Enlarged view of the region of multistability located at $\bar{\eta}<0$ in Fig. 6. Black line: Hopf bifurcation (subcritical). Green lines: saddle-node bifurcations. In the dark shaded region, only the quiescent, low activity state is stable. In the light shaded region, incoherence coexists with a collective oscillatory state-self-sustained due to recurrent excitation. In the brown region the low activity fixed point coexists with a high activity fixed point and with the oscillatory state. In the small dark purple region only the two high and low activity fixed points are attracting. Right panels: Sketches of the Poincaré section in different regions (assuming that it coincides with the one-dimensional manifold that connects different fixed points). The thick lines indicate two-dimensional manifolds, and periodic orbits are indicated by a point surrounded by a small circle. 

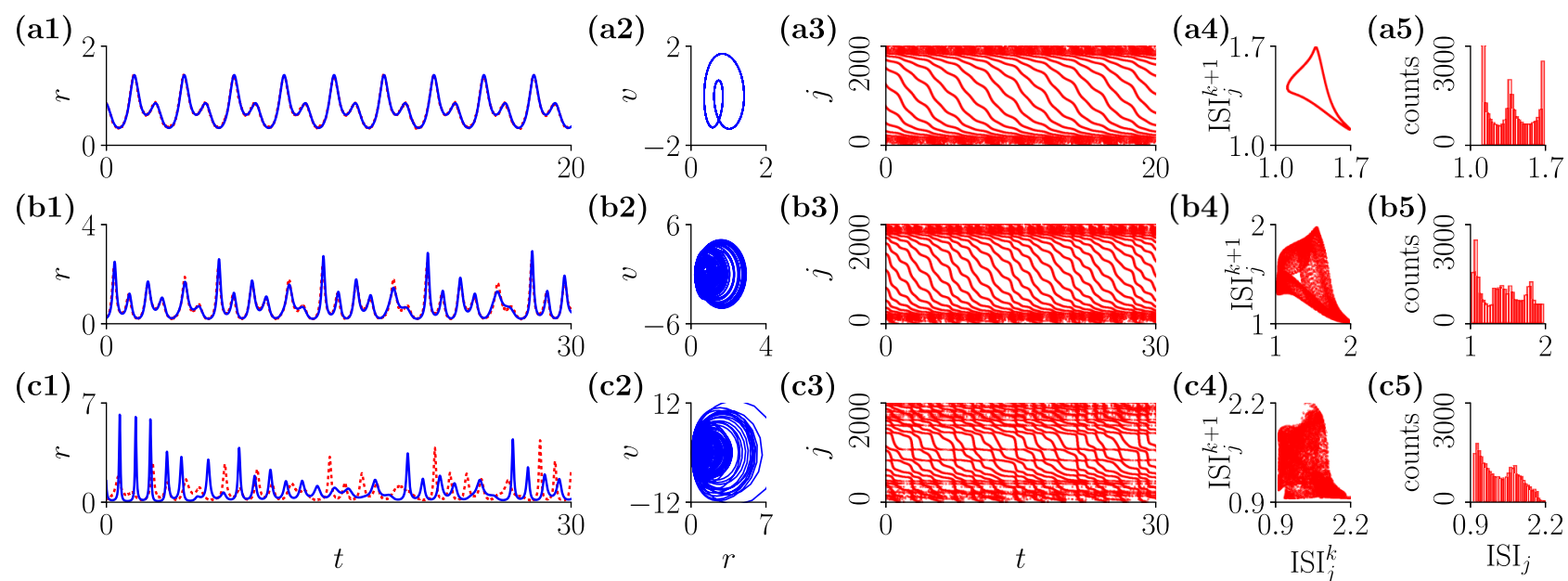

(b2)

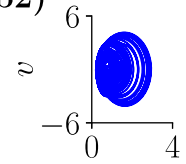

(c2)

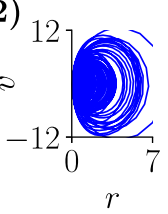

(b3)

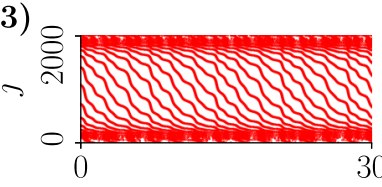

(b4)

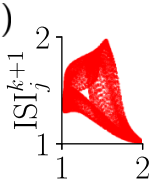

(b5)

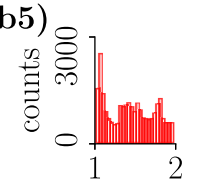

(c3)

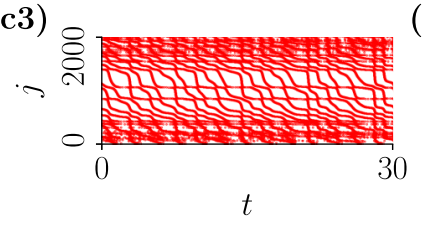

$(\mathbf{c 4})$

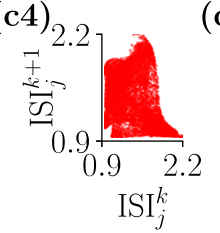

(c5)

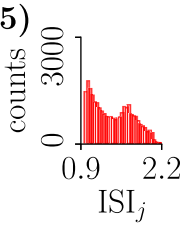

FIG. 8. Macroscopic (columns 1 and 2) and microscopic (columns 3-5) dynamics of (row a) PS-II states, (row b) M-PS states, and (row c) collective chaos for heterogeneous neurons-see Fig. 2 and Table I. Column 1: Time series of the firing rate for the FRE Eqs. (9) (blue) and for a population of $N=2000$ QIF neurons Eqs. (1) (red dotted). Column 2 shows the corresponding attractors, obtained using the FRE. In rows (a) and (b), the dynamics is periodic but, in contrast with the identical case, here the limit cycle is asymmetric due to the presence heterogeneity. Column 3 shows the raster plots corresponding to numerical simulations of a population of $N=2000$ QIF neurons Eqs. (1), and columns 4 and 5 show the corresponding return plots and ISI histograms, respectively. In the raster plots, each neuron index $j$ corresponds to a specific $\eta_{j}$ value (see text). For the computation of return plots and ISI histograms we used neuron $j=500$. In panels (a4) and (b4) one can see that the neuron behaves quasiperiodically, with two and three incommensurable frequencies, respectively. Note also the three peaks in panel (a5) due to the asymmetry of the limit cycle. In all panels we use $\Delta=0.1, \sqrt{\bar{\eta}}=3.5$, and (row a) $J=-9.60$; (row b) $J=-10.70$; (row c) $J=-11.30$.

a population of QIF neurons. All the three states are clearly reminiscent of the QPS, M-QPS and collective chaos states for identical neurons. In the columns (3-5) of Fig. 8 we also show the raster plots of the spiking activity of the population of QIF neurons together with the return plots and ISI histograms of a single neuron of the population. Due to the presence of heterogeneity, in the PS-II state neurons can be either periodic or two-frequency quasiperiodic, while in the M-PS they can be two- or three-frequency quasiperiodic, see Table I. The illustrative neuron chosen to plot the return maps and ISI histograms of Fig. 8 are, respectively, two-frequency and three-frequency quasiperiodic for Figs. 8(a4), 8(a5) and 8(b4), 8(b5). Note how, as in the QPS-asym in Fig. 2(f), the histogram of ISIs for a quasiperiodic neuron in the PS-II state has three peaks, due to the asymmetric shape of the limit cycle.

To further characterize the microscopic dynamics of PSII, M-PS and collective chaos, in Fig. 9 we calculate the time-averaged coupling-modified ISIs of the neurons, and plot them against each neuron natural ISI $T_{j}$. In the PS-II state shown in Fig. 9(a), the lower and upper plateaus correspond, respectively, to the average period between two consecutive peaks of the mean field, and to the period of the mean field oscillation in Fig. 8. Here it is convenient to recall Table I, where the relations between macroscopic and microscopic dynamics are indicated.

Finally, we investigate the bifurcations that connect these partially synchronous states, again relying on the computation of the Lyapunov spectrum of Eqs. (9). As we did in Section IV for identical neurons, we evaluate the four largest Lyapunov exponents along the $J$ direction in the phase diagrams, near the Hopf bifurcation. Figure 10 reveals a scenario qualitatively similar to the identical case (except that, at least for the specific $\bar{\eta}$ value adopted, no bistability was detected).
Starting from a fixed point, the Hopf bifurcation produces a periodic solution (PS-II) with a vanishing largest LE, which then undergoes a Neimark-Sacker bifurcation leading to a quasiperiodic solution (M-PS). Eventually, this quasiperiodic solution breaks down giving rise to a chaotic state. Finally, increasing inhibition above a critical level makes the Lyapunov exponents to change abruptly, and chaos is suddenly replaced by a periodic orbit (PS-I). This is in consistency with an exterior crisis undergone by the chaotic attractor.

\section{Boundaries of incoherence for large heterogeneity}

At this point, we discussed a fixed value of the heterogeneity $\Delta=0.1$. We now study the effect of increasing values of $\Delta$ on the stability boundaries of incoherence. As previ-
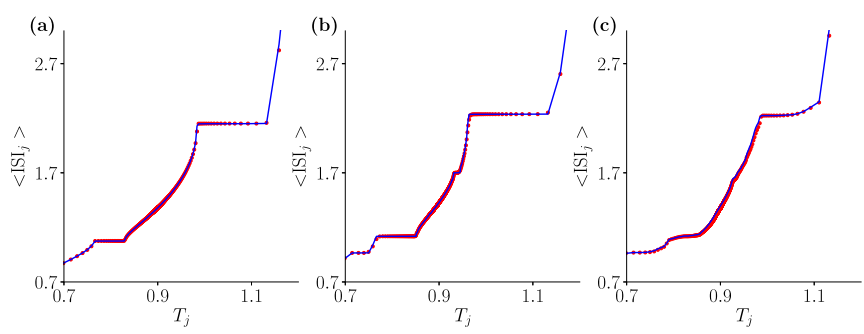

FIG. 9. Time-averaged coupling-modified ISIs as a function of the intrinsic ISI for a population of 2000 QIF neurons in three different states: (a) PS-II, (b) M-PS, and (c) collective chaos. The red dots are obtained with direct simulations of the population of QIF neurons, while the blue line is obtained forcing each neuron with the FRE. Note the multiple plateaus in the middle panel. Parameters are as in Fig. 8: $\sqrt{\bar{\eta}}=3.5$ and (a) $J=-9.60$; (b) $J=-10.70$; (c) $J=-11.30$. 


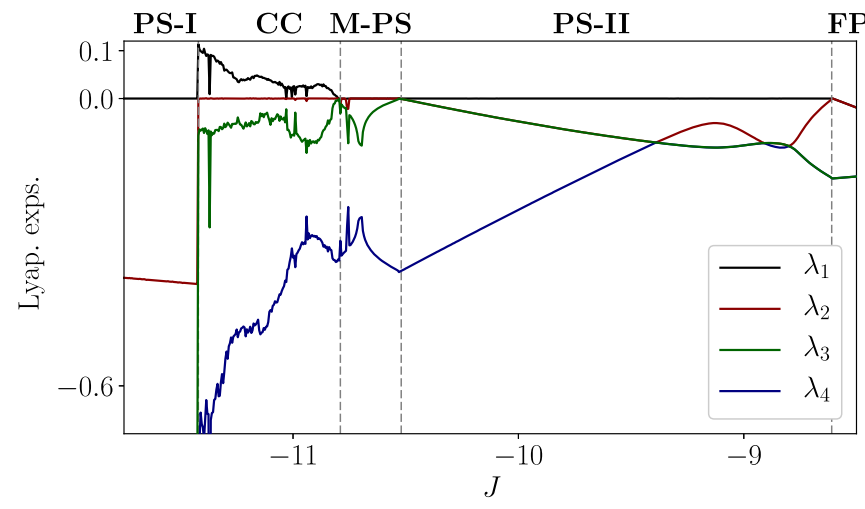

FIG. 10. The four largest Lyapunov exponents for $\Delta=0.1$ and $\sqrt{\bar{\eta}}=3.5$. The stability regions of the different attractors are indicated by vertical gray dashed lines.

ously discussed, the Hopf bifurcations become increasingly supercritical as the level of heterogeneity grows, and this is particularly pronounced for inhibitory coupling. Hence, the Hopf boundaries are a good proxy to bound the regions with oscillations of either type (PS-I, PS-II, M-PS, collective chaos).

Figure 11 shows the Hopf boundaries increasing values of $\Delta$. Note that the region of oscillations for inhibitory coupling progressively shrinks, and eventually disappears from the diagram. Accordingly, given a value of $\bar{\eta}$, there is a value of $\Delta$ for which, no matter how strong inhibition is, the neurons will not synchronize. The fragility of the oscillations against heterogeneity is consistent with previous computational studies of networks of inhibitory, conductance-based spiking neurons [77-79]. However, note that synchronization can always be

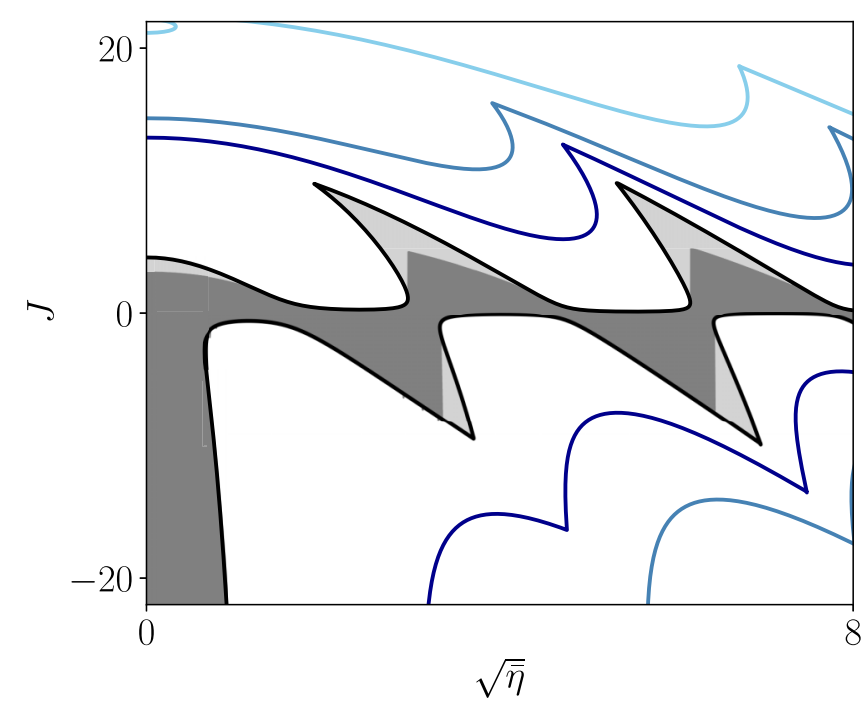

FIG. 11. Increasing the level of heterogeneity $\Delta$ reveals different synchronization scenarios for excitation and inhibition (see text). Black, dark blue, blue and light blue lines correspond, respectively, to the Hopf boundaries of Eqs. (9) with $\Delta=0.1,5,10,20$. These boundaries determine the regions of stability of the incoherent/asynchronous states. In the shaded regions incoherence is stable for $\Delta=0.1$. In the dark shaded region the only attractor is incoherence. achieved for strong enough excitatory coupling. This highlights a fundamental asymmetry between the excitatory and the inhibitory oscillatory regions in networks of QIF neurons. We emphasize that this asymmetric behavior is not found in the heterogeneous Kuramoto model with delay [64-66]. A possible explanation for such asymmetry is that, at variance with other self-sustained oscillators, QIF neurons cease to oscillate for strong enough inhibition. On the contrary, excitation just speeds up QIF neurons, which remain oscillatory.

\section{CONCLUSIONS AND DISCUSSION}

We analyzed the dynamics of a large population of QIF neurons with synaptic delays. To a large extent the analysis was carried out using the FRE Eqs. (9), which is mathematically tractable and allows for an efficient computational analysis. For identical neurons, we have extended the analytical results in Ref. [28] to the excitable regime $(\bar{\eta}<0)$. Our analytical predictions pointed out parameter regimes where nontrivial dynamics should necessarily occur. In these regions of parameters we performed an extensive numerical exploration supported by the computation of the Lyapunov spectrum, which revealed the existence of partially synchronous states. One of these states, which we called M-QPS, appears after a Neimark-Sacker bifurcation of QPS that superimposes a second (modulating) frequency. Partially synchronous states-especially QPS — coexist with full synchronization in a large region of the parameter space. The existence in the phase diagram Fig. 5 of what looks like a second tongue for QPS is an intriguing finding of this work. Can its origin be understood, at least heuristically? We finally showed that the partially synchronized states observed in the absence of disorder also have their counterpart in the presence of heterogeneity. However, disorder induces diversity in the microscopic behaviors of the single neurons.

To conclude, we demonstrate that most of the dynamics of the FRE Eqs. (9) investigated here cannot be reproduced using traditional firing rate models [2-5]. To show this we note that the fixed points of Eqs. (9) have precisely the structure of traditional firing rate models, while the dynamics is generically different [55]. Solving the fixed point equation corresponding to Eq. (9a) for $v$, and substituting it into the fixed point equation corresponding to Eq. (9b), one obtains an equation for the steady firing rate,

$$
r_{*}=\Phi\left(J r_{*}+\bar{\eta}\right)
$$

The function

$$
\Phi(x)=\frac{1}{\sqrt{2} \pi} \sqrt{x+\sqrt{x^{2}+\Delta^{2}}}
$$

is the so-called "transfer function" of a population of QIF neurons with Lorentzian distribution of currents $[49,55]-$ steady-state equations for arbitrary distributions of currents can be obtained self-consistently; see Eq. (C1) in Ref. [26]. Clearly, the traditional first-order firing rate model with time delays,

$$
\dot{r}=-r+\Phi\left(J r_{D=1}+\bar{\eta}\right)
$$

largely investigated in the literature, has exactly the same fixed points as Eqs. (9) but different dynamics-see, e.g., 


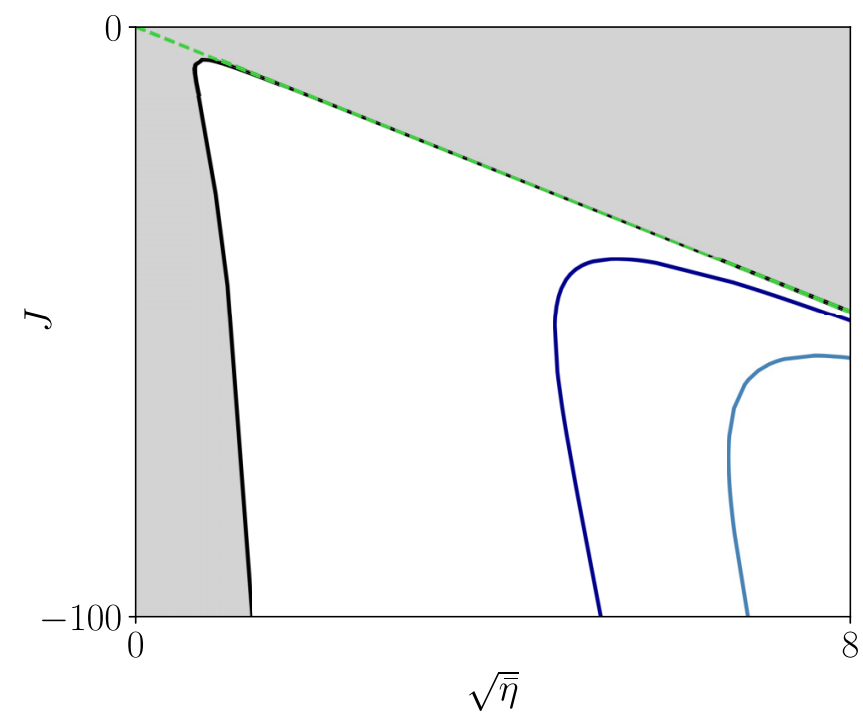

FIG. 12. Oscillations emerge only for inhibitory coupling in the traditional firing rate model Eq. (17). In the gray region, limited by the black line $(\Delta=0.1)$, the fixed point determined by Eq. (16) is stable and looses stability via a Hopf bifurcation-compare with Fig. 11. The dark blue and blue curves correspond to $\Delta=5$ and 10 , respectively. The green dashed boundary corresponds to the case $\Delta=0$ and is a straight line.

Refs. [7-13] for studies of Eqs. (17) using different transfer functions. Indeed, the linear stabililty analysis of the fixed points of Eq. (17) gives the characteristic equation

$$
\lambda=-1+\Phi^{\prime} J e^{-\lambda},
$$

where $\lambda$ is an eigenvalue, and $\Phi^{\prime}$ is the derivative of the transfer function evaluated at the fixed point $r_{*}$, determined by Eq. (16). The nonstationary instabilities (obtained using the condition of marginal stability $\lambda=i \Omega$ ) are depicted in Fig. 12 for different values of the heterogeneity $\Delta$, and clearly differ from the Hopf boundaries of the FRE Eqs. (9) shown in Fig 11. Specifically, the traditional firing rate model Eq. (17) only displays oscillations for inhibitory coupling and $\bar{\eta}>0$, while the FRE Eqs. (9) show oscillations for both excitation and inhibition, even for $\bar{\eta}<0$-see Figs. 1, 6, and 11. Moreover, the tent-shaped structure of the Hopf boundaries of Eqs. (17) is lost in the traditional firing rate model Eq. (17).

Nonetheless, note that as the heterogeneity $\Delta$ is increased, the behavior of the Hopf boundaries of Eq. (17) qualitatively agrees with that of the FRE Eqs. (9): The region of oscillations in both models shifts to large $\bar{\eta}$ values, in consonance with the well known result that quenched heterogeneity cannot be counterbalanced by inhibitory coupling to produce synchronization [55,77-79]. Moreover, we have shown that for large heterogeneity the Hopf boundaries of Eqs. (9) become supercritical, and this coincides with what is generically found in traditional firing rate models with small delays [10]. In fact, though Eq. (17) is heuristic, it has proven to be remarkably effective at describing the oscillatory dynamics of networks of spiking neurons with strong noise [7-14], and is a paradigmatic mean-field model to investigate the effect of various types of delays in neuronal networks; see, e.g., Refs. [15-25].

Finally, we want to note the resemblance of the partially synchronized states investigated here with the so-called sparsely synchronized states [9], in which strong inhibition and noise produce irregular spiking but a coherent macroscopic oscillation. Remarkably, in both states the period of the macroscopic oscillation is determined by the time delay but differs from the ISIs of the single cells. However, microscopically, the neurons have a qualitatively different behavior: In the QPS, their dynamics is purely deterministic and quasiperiodic, while in the sparse synchrony it is stochastic and irregular.

\section{ACKNOWLEDGMENTS}

We acknowledge support by the Spanish Ministry of Economy and Competitiveness under Projects No. FIS201674957-P, No. PSI2016-75688-P, and No. PCIN-2015-127. We also acknowledge support by the European Union's Horizon 2020 Research and Innovation programme under the Marie Skłodowska-Curie Grant No. 642563.
[1] A. L. Hodgkin and A. F. Huxley, J. Phys. 117, 500 (1952).

[2] H. R. Wilson and J. D. Cowan, Biophys. J. 12, 1 (1972).

[3] P. Dayan and L. F. Abbott, Theoretical Neuroscience (MIT Press, Cambridge, MA, 2001).

[4] W. Gerstner and W. M. Kistler, Spiking Neuron Models: Single Neurons, Populations, Plasticity (Cambridge University Press, Cambridge, 2002).

[5] G. B. Ermentrout and D. H. Terman, Mathematical Foundations of Neuroscience (Springer, Berlin, 2010), Vol. 64.

[6] P. Ashwin, S. Coombes, and R. Nicks, J. Math. Neurosci. 6, 2 (2016).

[7] A. Roxin, N. Brunel, and D. Hansel, Phys. Rev. Lett. 94, 238103 (2005).

[8] D. Battaglia, N. Brunel, and D. Hansel, Phys. Rev. Lett. 99, 238106 (2007).
[9] N. Brunel and V. Hakim, Chaos: Interdisc. J. Nonlin. Sci. 18, 015113 (2008).

[10] A. Roxin and E. Montbrió, Physica D 240, 323 (2011).

[11] E. Ledoux and N. Brunel, Front. Comput. Neurosci. 5, 25 (2011).

[12] S. Keeley, A. A. Fenton, and J. Rinzel, J. Neurophysiol. 117, 950 (2017).

[13] C. Kim, U. Egert, and A. Kumar, bioRxiv (2018), doi: 10.1101/360479.

[14] J. Senk et al., arXiv:1801.06046 (2018).

[15] A. Hutt and F. M. Atay, Phys. Rev. E 73, 021906 (2006).

[16] P. C. Bressloff and Z. P. Kilpatrick, Phys. Rev. E 78, 041916 (2008).

[17] N. Venkov, S. Coombes, and P. Matthews, Physica D: Nonlin. Phenom. 232, 1 (2007). 
[18] S. Coombes and C. Laing, Philos. Trans. Roy. Soc. London A: Math., Phys. Eng. Sci. 367, 1117 (2009).

[19] G. Faye and O. Faugeras, Physica D: Nonlin. Phenom. 239, 561 (2010).

[20] J. Touboul, Physica D: Nonlin. Phenom. 241, 1223 (2012).

[21] M. T. Wilson, P. A. Robinson, B. O'Neill, and D. A. Steyn-Ross, PLoS Comput. Biol. 8(6), e1002560 (2012).

[22] R. Veltz, SIAM J. Appl. Dynam. Syst. 12, 1566 (2013).

[23] R. Veltz and O. Faugeras, SIAM J. Math. Anal. 45, 1527 (2013).

[24] G. Faye and J. Touboul, SIAM J. Appl. Math. 74, 1657 (2014).

[25] K. Dijkstra et al., Physica D: Nonlin. Phenom. 297, 88 (2015).

[26] E. Montbrió, D. Pazó, and A. Roxin, Phys. Rev. X 5, 021028 (2015).

[27] To be consistent with previous work investigating the collective dynamics of identical oscillators-see Ref. [80] and references therein-we adopt the term "partial synchronization" to refer to states which are neither fully synchronous nor asynchronous (excluding cluster states). We note that the same term is used to designate synchronous states in the Kuramoto model with heterogeneity. Here we use the same terminology for both types of states (see Sec. V).

[28] D. Pazó and E. Montbrió, Phys. Rev. Lett. 116, 238101 (2016).

[29] N. Nakagawa and Y. Kuramoto, Physica D 80, 307 (1995).

[30] C. van Vreeswijk, Phys. Rev. E 54, 5522 (1996).

[31] A. Vilfan and T. Duke, Phys. Rev. Lett. 91, 114101 (2003).

[32] P. K. Mohanty and A. Politi, J. Phys. A: Math. Gen. 39, L415 (2006).

[33] S. Olmi, A. Politi, and A. Torcini, Europhys. Lett. 92, 60007 (2010).

[34] S. Luccioli, S. Olmi, A. Politi, and A. Torcini, Phys. Rev. Lett. 109, 138103 (2012).

[35] M. Rosenblum and A. Pikovsky, Phys. Rev. Lett. 98, 064101 (2007).

[36] R. Burioni, S. di Santo, M. di Volo, and A. Vezzani, Phys. Rev. E 90, 042918 (2014).

[37] A. A. Temirbayev, Z. Z. Zhanabaev, S. B. Tarasov, V. I. Ponomarenko, and M. Rosenblum, Phys. Rev. E 85, 015204(R) (2012).

[38] A. A. Temirbayev, Y. D. Nalibayev, Z. Z. Zhanabaev, V. I. Ponomarenko, and M. Rosenblum, Phys. Rev. E 87, 062917 (2013).

[39] A. Politi and M. Rosenblum, Phys. Rev. E 91, 042916 (2015).

[40] P. Clusella, A. Politi, and M. Rosenblum, New J. Phys. 18, 093037 (2016).

[41] M. Rosenblum and A. Pikovsky, Phys. Rev. E 92, 012919 (2015).

[42] E. M. Izhikevich, Dynamical Systems in Neuroscience (MIT Press, Cambridge, Massachusetts, 2007).

[43] For the numerical simulations of the population of QIF neurons we use the Euler method with time step $\delta t=10^{-5}$. For the integration of the FRE Eqs. (9) we use a third-order AdamsBashforth-Moulton predictor-corrector scheme with a timestep $\delta t=10^{-4}$ [81]. In all simulations shown, initial transients were discarded.

[44] B. Pietras and A. Daffertshofer, Chaos 26, 103101 (2016).

[45] E. Ott and T. M. Antonsen, Chaos 18, 037113 (2008).

[46] D. Pazó and E. Montbrió, Phys. Rev. X 4, 011009 (2014).

[47] T. B. Luke, E. Barreto, and P. So, Neural Comput. 25, 3207 (2013).
[48] P. So, T. B. Luke, and E. Barreto, Physica D 267, 16 (2014).

[49] C. R. Laing, Phys. Rev. E 90, 010901 (2014).

[50] C. R. Laing, SIAM J. App. Dynam. Syst. 14, 1899 (2015).

[51] S. Coombes and Á. Byrne, in Nonlinear Dynamics in Computational Neuroscience, edited by F. Corinto and A. Torcini (Springer International Publishing, Cham, 2019), pp. 1-16.

[52] J. Roulet and G. B. Mindlin, Chaos: Interdisc. J. Nonlin. Sci. 26, 093104 (2016).

[53] B. Ermentrout and N. Kopell, SIAM J. Appl. Math. 46, 233 (1986).

[54] I. Ratas and K. Pyragas, Phys. Rev. E 94, 032215 (2016).

[55] F. Devalle, A. Roxin, and E. Montbrió, PLoS Comput. Biol. 13(12), e1005881 (2017).

[56] I. Ratas and K. Pyragas, Phys. Rev. E 96, 042212 (2017).

[57] G. Dumont, G. B. Ermentrout, and B. Gutkin, Phys. Rev. E 96, 042311 (2017).

[58] H. Schmidt, D. Avitabile, E. Montbrió, and A. Roxin, PLoS Comput. Biol. 14(9), e1006430 (2018).

[59] J. M. Esnaola-Acebes, A. Roxin, D. Avitabile, and E. Montbrió, Phys. Rev. E 96, 052407 (2017).

[60] C. R. Laing, J. Math. Neurosci. 8, 4 (2018).

[61] M. di Volo and A. Torcini, Phys. Rev. Lett. 121, 128301 (2018).

[62] M. K. Stephen Yeung and S. H. Strogatz, Phys. Rev. Lett. 82, 648 (1999).

[63] M. Y. Choi, H. J. Kim, D. Kim, and H. Hong, Phys. Rev. E 61, 371 (2000).

[64] M. G. Earl and S. H. Strogatz, Phys. Rev. E 67, 036204 (2003).

[65] E. Montbrió, D. Pazó, and J. Schmidt, Phys. Rev. E 74, 056201 (2006).

[66] W. S. Lee, E. Ott, and T. M. Antonsen, Phys. Rev. Lett. 103, 044101 (2009).

[67] S. Watanabe and S. H. Strogatz, Physica D 74, 197 (1994).

[68] A. Pikovsky and M. Rosenblum, Physica D 240, 872 (2011).

[69] I. V. Tyulkina, D. S. Goldobin, L. S. Klimenko, and A. Pikovsky, Phys. Rev. Lett. 120, 264101 (2018).

[70] E. Montbrió and D. Pazó, Phys. Rev. Lett. 120, 244101 (2018).

[71] P. Clusella and A. Politi, arXiv:1810.01281.

[72] J. D. Farmer, Physica D 4, 366 (1982).

[73] J. H. Curry and J. A. Yorke, The Structure of Attractors in Dynamical Systems, No. 668 in Springer Notes in Mathematics (Springer-Verlag, Berlin, 1978), pp. 48-56.

[74] J. L. Kaplan and J. A. Yorke, in Functional Differential Equations and Approximation of Fixed Points, Vol. 730 of Lecture Notes in Mathematics, edited by H. O. Walter and H.-O. Peitgen (Springer-Verlag, Berlin, 1979), pp. 204-227.

[75] Actually, we have not explored the region close to the supercritical bifurcation just above $\sqrt{\bar{\eta}}=2 \pi$.

[76] The degenerate point is a codimension-three point because the instability for $n=1$ is degenerate exactly at that point (see the Supplemental Material of Ref. [28]).

[77] X.-J. Wang and G. Buzsáki, J. Neurosci. 16, 6402 (1996).

[78] J. A. White et al., J. Comput. Neurosci. 5, 5 (1998).

[79] P. Tiesinga and J. V. José, Netw., Comput. Neural Syst. 11, 1 (2000).

[80] A. Pikovsky and M. Rosenblum, Chaos 25, 097616 (2015).

[81] W. H. Press, S. A. Teukolsky, W. T. Vetterling, and B. P. Flannery, Numerical Recipes in FORTRAN (2nd ed.): The Art of Scientific Computing (Cambridge University Press, New York, NY, 1992). 